\title{
The Effect of Variable Wake Up Time on the Utilization of Sleep Modes in Femtocell Mobile Access Networks
}

\author{
Willem Vereecken *, Ivaylo Haratcherev ${ }^{\dagger}$, Margot Deruyck*, Wout Joseph*, Mario Pickavet*, \\ Luc Martens* and Piet Demeester* \\ ${ }^{*}$ Ghent University - IBBT, Department of Information Technology (INTEC) \\ Gaston Crommenlaan 8, Bus 201, 9050 Ghent, Belgium \\ email: Willem.Vereecken@INTEC.ugent.be \\ web: www.INTEC.ugent.be \\ $\dagger$ Alcatel-Lucent Bell Labs France, Networking and Networks Domain \\ Centre de Villarceaux, Route de Villejust, 91260 Nozay, France
}

\begin{abstract}
Femtocells are considered as an enabler for low power, high bit rate future mobile access networks. In this study, we look at them as a technology to cover an area with a high bit rate connectivity. From the evaluation it is clear that sleep modes are imperative to maximise the energy efficiency of the mobile access network. We evaluate the power reduction and wake up time of different sleep modes and apply them to the model in order to evaluate the influence of the power consumption of the mobile access network. We demonstrate that fast wake up times and low power sleep modes are essential in order to make femtocells a viable technology for mobile access networks.

Index Terms-Network Power Consumption, Carbon Footprint, Mobile Access Networks, Femtocells, Energy Reduction, Green ICT
\end{abstract}

\section{INTRODUCTION}

Energy efficiency is a growing concern in the modern day telecommunication industry. Increasing energy prices on the one hand and growing attention for environmental aspects such as climate change and the associated carbon emissions lead to a trend in which the energy consumption of technologies needs to be reduced. In the current network technologies, access networks are largely consuming most of the energy. Of these access networks, high bit rate mobile access networks are a concern [1].

Also, the bit rate demand of mobile users is increasing in order to enable applications like gaming or video streaming [2]. This high bit rate demand will only be available to the user at shorter distances to the base stations and assuring these bit rates will require more dense mobile access networks. Since it will be difficult to achieve these densities with large base stations or so called macrocells, a new technology called femtocells is being examined.

Femtocells are inexpensive, low RF power base stations with only a small coverage area and associated reduced power consumption. On the other hand, covering an area will require much more devices. If we want to make this kind of access network energy efficient, the introduction of sleep modes will become imperative. In this study we investigate the feasibility of the introduction of sleep modes in femtocell access networks and we evaluate the options of different sleep modes and their associated wake up times.

\section{SLEeP MOdES IN Femtocells}

The largest part of the power consumed in a femtocell is related to the RF front-end (45\%) and the TCXO heater (7\%). Hence, switching these components off reduces the consumed power by more than $50 \%$. At the same time, waking up the $\mathrm{RF}$ is in the order of few hundred of miliseconds. The TCXO will indeed take some time to heat back up, but our tests show that apart from some induced clock drift, there will be no disruption of femtocell operation.

Based on test results and simulation, we defined some power-save modes, ordered by 'depth'. The deeper a sleep mode is the more power is saved, but the more the cost of that mode is - i.e. it takes the femtocell additional time to wake-up.

- On: The femtocell is in full operation, and is consuming maximum power. Depending on the design that power is typically between 8 and 15 Watts.

- Stand-by: The femtocell is in 'light' sleep and can wakeup quickly. The RF and the TCXO heater are switched off.

- Sleep: The femtocell is in 'deep' sleep and needs some time to wake up. In this mode only the power supply, the backend connection and the generic CPU core remain active.

- Offline: The femtocell is off and consumes no power.

These wake-up times are summarized in Table I. The power consumption is expressed as a percentage of the active power consumption. 


\begin{tabular}{lcc}
\hline Mode & Wake-up time (s) & Power Consumption \\
\hline On & N/A & $100 \%$ \\
Stand-by & 0.5 & $50 \%$ \\
Sleep & 10 & $15 \%$ \\
Offline & 30 & \pm 0
\end{tabular}

Table I: Wake up times of femtocells in different modes

\begin{tabular}{ccc}
\hline Coding Scheme: & Bit Rate (Mbps) & Range $(\mathrm{m})$ \\
\hline 1/4 QPSK & 1.3 & 51.4 \\
1/2 QPSK & 2.5 & 39.5 \\
3/4 QPSK & 3.8 & 30.1 \\
1/2 16-QAM & 5 & 24.3 \\
3/4 8-QAM & 5.6 & 22.2 \\
3/4 16-QAM & 7.5 & 18.1 \\
3/4 64-QAM & 11.3 & 11 \\
\hline
\end{tabular}

Table II: Ranges of HSPA

\section{HSPA AND PATH LOSS}

HSPA (High Speed Packet Access) is currently widely introduced in operator networks [5]. It works in the $2.1 \mathrm{GHz}$ band but a higher performance is obtained by using improved coding schemes and refined protocols for communication between handset and base station. HSPA provides peak data rates up to $14 \mathrm{Mbps}$ in the downlink. In the downlink (DL), HSPA supports different coding schemes each corresponding with a certain bit rate and a certain range. Each coding scheme consists of a modulation scheme which translates the binary bit stream into an analogue signal, and a coding rate, which indicates how many redundant bits will be added per number of information bits. Based on the used modulation scheme and coding rate it is possible to determine the bit rate as shown in Table II. For the uplink (UL), HSPA supports only one modulation scheme (i.e., BPSK) resulting in a bit rate of 1.5 Mbps.

Each coding scheme also corresponds with a certain receiver SNR (Signal-to-Noise Ratio) which represents the SNR at the receiver for a certain BER (Bit Error Rate). This receiver SNR is taken into account when determining the maximum allowable path loss $P L_{\max }$ to which a transmitted signal can be subjected while still being detectable at the receiver. The path loss is the ratio of the transmitted power to the received power. Once $P L_{\max }$ is known, the corresponding range can be calculated by using a propagation model. Here, the ITU-R P.1238 model for a residential environment is used as this is the most appropriate model for femtocell base stations and the environment considered. [6].

It is thus clear that the coding scheme influences both the bit rate and the range.

\section{Model of a Femtocell Access Network}

\section{A. Covering an area for a certain bit rate}

When designing an access network, the principal question is how to provide the user with a certain access bit rate. In mobile access networks, the user can be located at any place in a certain area. The base stations are then used to cover this area, assuring that a user is able to connect to the access network at any place in the covered area.

As we explained in section III, the highest bit rates are available closest to a base station. This implies that the main driving parameter for the coverage design is the bit rate we want to guarantee for a user.

In this study, we assume the influence of the distance of the user and the provided bit rate on the power consumption of the base station to be marginal. Hence, an active femtocell will have a fixed power consumption. This means the overall power consumption of the access network (without sleep modes) is proportional to the assumed base station density. When we want to cover a certain area, we know that a hexagonal grid provides the most efficient coverage. Hence, in this study, we assume a hexagonal grid covering a certain area.

\section{B. Introducing Sleep Modes}

When we calculate the base station density when covering for the highest bit rate of HSPA, we get a density of 3181 base stations per $\mathrm{km}^{2}$. In comparison, in most countries, an area is considered to be densely populated as of approximately 500 people per $\mathrm{km}^{2}$. Moreover, it is safe to assume that only a limited number of people will require the highest bit rates.

Therefore, when one wants to use femtocells to cover a certain area, the use of sleep modes will be indispensible. In [7] we derived a heuristic to establish the least number of active base stations required for a given user distribution with a certain bit rate demand in a given mobile access network. The principle of the heuristic is to gradually switch on base stations satisfying as many users as possible in each step. The effectiveness of the heuristic is based on the fact that, although the network is designed to cover for a high bit rate, in reality there will be many users requiring lower bit rates, thus being able to connect to a base station further away than the nearest base station. It is important to note that the heuristic is intended as a means to establish a lower bound for the potential of sleep modes. The usability in practical situations is impaired by the requirement of exact knowlegde of a users location.

An example distribution is displayed in Fig. 1. In an access network with 1951 base stations users were distributed with a user density of 1000 users per $\mathrm{km}^{2}$. For the bit rate requirements, we assumed an exponential distribution based on the available bit rates:

$$
\phi(B R) \propto \frac{1}{B R^{\alpha}}
$$

The factor $\alpha$ is determined so that $10 \%$ of the users require a bit rate higher than 5 Mbps. This corresponds with the assumption that most users require lower bit rates.

We can see in the example that 118 of the 1951 base stations are active. We denote this as the active base station fraction $F_{A}=6.0 \%$. Typically the base stations closes to the users with high demand are switched on and already provide a good coverage for the users with lower requirements. Additionally, some coverage holes for low bit rates need to be eliminated, leading to the optimal active base station distribution. 


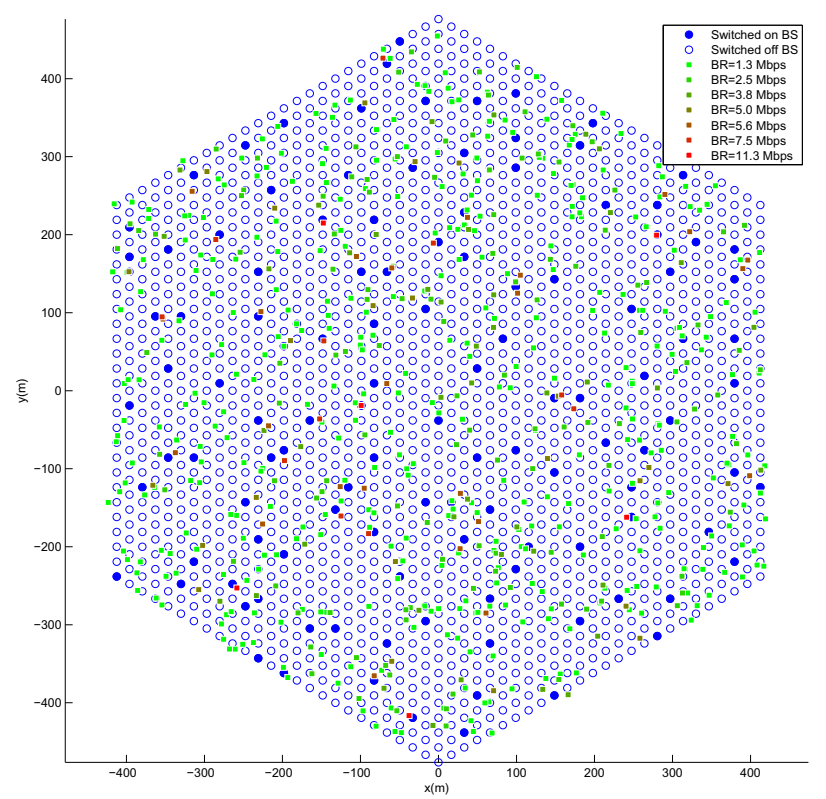

Figure 1: Example of the Introduction of Sleep Modes in an HSPA Femtocell Access Network with Static users. $\left(D_{U}=\right.$ $1000, P_{>5 \text { Mbps }}=10 \%$ )

\section{The Influence of Wake Up Times}

The calculation of the optimal distribution of active femtocells does not take into account any time constraints. If waking up and handing over to a different cell would happen instantaniously, this would not matter. The optimal distribution can than be applied at any point in time and will be closely correlated to the one at the previous point in time. However, as we already demonstrated in section II, there are different possible sleep modes, each requiring a certain wake up time.

A first factor to take into account is the moving speed of the users. If a user moves out of range of a cell before the next cell can wake up, there is a problem. As such, we need to make sure the the neighbouring cells are in the appropriate sleep mode. Hence, we define a maximal speed of a user in the network $v_{\max }$. In this study, we vary this $v_{\max }$ between 0 and $3 \mathrm{~m} / \mathrm{s}$, corresponding to the speed of a running person.

Based on this speed, first of all, we need a denser grid as the user cannot move out of range of a base station before the next cell can wake up from stand-by. The distance he can travel during this time of a few hundreds of miliseconds needs to be taken from the cell size.

Additionally, based on the distance a user can travel in order to wake up from sleep or off mode, the right sleep mode needs to be defined for neighbouring cells.

A different reason why a femtocell could have to change its operating mode is when a user shifts from a low bit rate requirement to a high bit rate requirement. Again, this can be related to the speed with which a user changes its behavior. This change is however much more difficult to cover for. The main empowering factor of the sleep mode introduction is the fact that there is a limited amount of users requiring a high

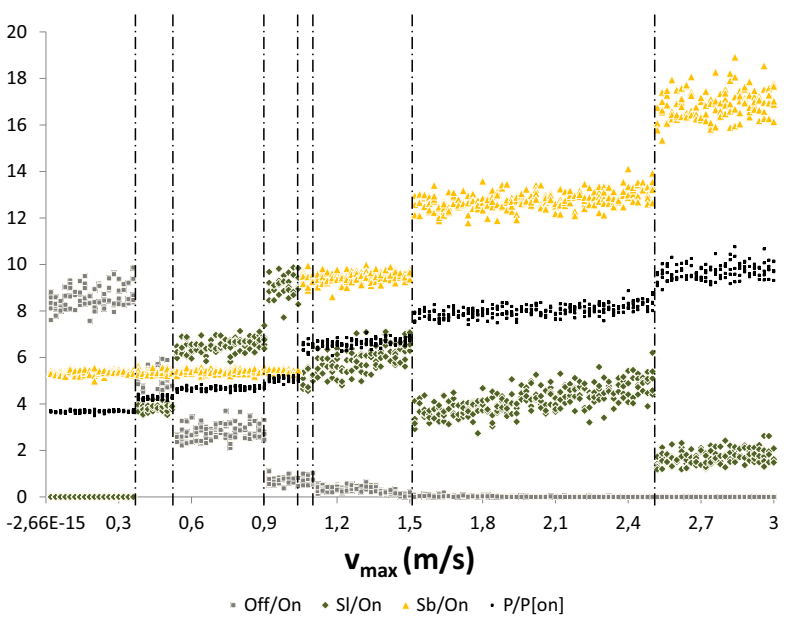

Figure 2: Influence of the Maximal Allowed User Speed on the Number of Base Stations in Offline, Stand-by or Sleep Mode and the Power Consumption of the Mobile Access Network

bit rate. If we assume that at any point in time any user might need a high bit rate and needs to obtain it instantaniously, we need to treat him like a high bit rate requiring user.

On the other hand, the case where a user switches from the lowest bit rate to the highest available is the most extreme case and will occur only exceptionally. Moreover, with other users being active in the network, there is a high probability the user will be able to connect to a base station with a higher bit rate than the previously required. Combining this with the possibility to wake up femtocells within a reasonable amount of time, it is likely that this problem can be tackled without having to guarantee immediate access to the highest bit rates at all times. On the other hand, we consider this out of the scope of the study performed in this paper.

\section{Evaluation of THE MODEL}

\section{A. Influence of the Maximal Allowed User Speed $\left(v_{\max }\right)$}

We simulated active base station distributions $v_{\max }$ varying between 0 and $3 \mathrm{~m} / \mathrm{s}$ with a user density $\mathrm{D}_{\mathrm{U}}=1000$ users $/ \mathrm{km}^{2}$. The results are displayed in Fig. 2 . It is immediately clear that, due to the variation of active base stations and the variation in the distribution, the exact number of base stations in a certain mode will be difficult to predict. We can see from the fraction of offline base stations compared to online base stations, there are almost no offline base stations at $v_{\max }>1$. Hence, the entire mobile access network is filled with base stations in sleep mode.

\section{B. Influence of Online The Base Station Density $\left(D_{B}^{O n}\right)$}

The density of online base stations $\left(D_{B}^{O n}\right)$ has a large impact on the number of femtocells in stand-by or sleep mode. We simulated situations with user densities varying between 25 and 2000 users per $\mathrm{km}^{2}$. The result is displayed in Fig. 3. We displayed the density of offline, sleeping and stand-by base stations in function of the online base station density. Note that in case of 1000 users per $\mathrm{km}^{2}$ (cfr. section V-A) the online 

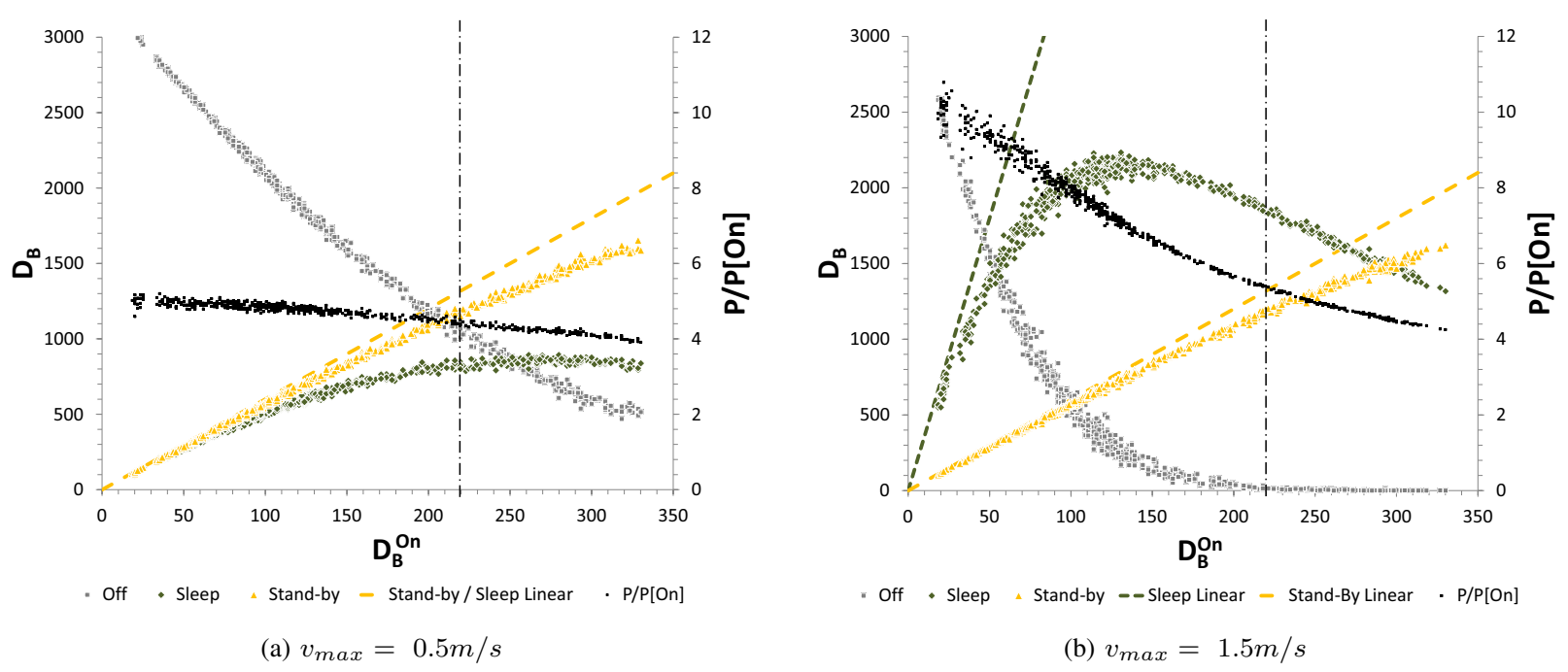

Figure 3: Influence of the Online Base Station Density $D_{B}^{O n}$ on the Base Station Density in Offline, Stand-by or Sleep Mode and the Power Consumption of the Mobile Access Network

base station density is about 215 base stations per $\mathrm{km}^{2}$, which is also indicated. Additionally, we displayed the relative power consumption.

In Fig. 3a we displayed the result when assuming a maximal user speed $v_{\max }=0.5 \mathrm{~m} / \mathrm{s}$. As of $D_{B}^{o n}>75$, the number of sleeping base stations begins to decrease from the linear trend due to overlaps of sleep mode zones in the network. As of $D_{B}^{o n}>125$, the number of base stations in stand-by mode also tends to decrease from the linear trend for the same reason. At these slow speeds, the power consumption of the total network is about $4-5$ times the power consumption of the active base station.

For higher speeds, for example $v_{\max }=1.5 \mathrm{~m} / \mathrm{s}$ (Fig. 3b), a higher number of base stations in sleep is required. Hence, the influence of the base station density starts at about $D_{B}^{o n} \approx 25$ . As of $D_{B}^{o n} \approx 125$, the network is so dense that the overlap causes the number of sleeping base stations to be reducing. When evaluating the power consumption, one sees that for low densities the power consumption is up to thirteen times the power consumption of an online base station. For higher densities this reduces to about five.

\section{CONCLusions}

Femtocells are a promising emerging technology for mobile access networks. Due to their small scale they consume less than a macrocell station but also cover a smaller area. As a consequence, it will be required to be able to introduce sleep modes in femtocell base stations in order to make a sustainable deployment possible.

Multiple sleep modes are available. We made a distinction between offline, sleeping and stand-by mode. The deeper the sleep mode, the lower the power consumption of the station, but also the longer it takes to bring the base station online.

It is demonstrated that the power consumption of these sleep modes has a large impact on the mobile access network power consumption. It is important to both reduce the power consumption of the sleep modes as well as increase the speed of taking a femtocell out of sleep mode in order to maximize the energy saving effect of sleep modes.

\section{ACKNOWLEDGEMENTS}

The research leading to these results has received funding from the European Union Seventh Framework Programme (FP7/2007-2013) under grant agreement n. 257740 (Network of Excellence "TREND") and the IBBT-project Green ICT.

W. Joseph is a Post-Doctoral Fellow of FWO-V (Research Foundation Flanders).

\section{REFERENCES}

[1] W. Vereecken, W. Van Heddeghem, M. Deruyck, B. Puype, B. Lannoo, W. Joseph, D. Colle, L. Martens, and P. Demeester, "Power consumption in telecommunication networks: overview and reduction strategies," Communications Magazine, IEEE, vol. 49, no. 6, pp. 62 -69, june 2011.

[2] Cisco VNI Mobile, "Global mobile data traffic forecast update 2010 2015," Cisco, Tech. Rep., 2011.

[3] G. Korinthios, E. Theodoropoulou, N. Marouda, I. Mesogiti, E. Nikolitsa, and G. Lyberopoulos, "Early experiences and lessons learned from femtocells," Communications Magazine, IEEE, vol. 47, no. 9, pp. 124 -130 , september 2009.

[4] 3rd Generation Partnership Project: Basestation (BS) radio transmission and reception (FDD) (Release 6), TS 25.104, 3GPP.

[5] 3rd Generation Partnership Project: Physical layer aspects of UTRA High Speed Downlink Packet Access (Release 4), TR 25.848 v4.0.0., 3GPP, March 2001.

[6] ITU-R, "Propagation data and prediction methods for the planning of indoor radiocommunication systems and radio local area networks in the frequency range $900 \mathrm{MHz}$ to $100 \mathrm{GHz}$," Recommendation P.1238-6, 2009.

[7] W. Vereecken, M. Deruyck, D. Colle, W. Joseph, M. Pickavet, L. Martens, and P. Demeester, "Evaluation of the potential for energy saving in macrocell and femtocell networks using a heuristic introducing sleep modes in base stations," submitted to EURASIP journal on wireless communications and networking, august 2011. 